\title{
On a generalization of the Maillet determinant II
}

\author{
by \\ Shigeru Kanemitsu (Fukuoka) and Takako Kuzumaki (Gifu)

\section{Dedicated to Professor Katsuya Miyake on his sixtieth birthday}

1. Introduction. In the previous paper [16] (referred to as Part I hereafter) we have rendered it visible that the two intimately connected problems of number theory, Maillet determinants and Chowla's problem, were dealt with in complete separation, save for Girstmair [11], where this interaction was duly noticed and proved to be crucial.

After Carlitz-Olson's remarkable 1955 discovery [3] of the expression of the Maillet determinant of prime modulus $p$ (introduced by E. Maillet [22] in 1913) in terms of the relative class number of the prime cyclotomic field $\mathbb{Q}\left(\zeta_{p}\right), \zeta_{p}$ a primitive $p$ th root of unity, there have appeared a number of papers concerning the relation between Maillet-type determinants of general modulus and relative class numbers of imaginary subfields of a cyclotomic field.

Chowla's problem was apparently to mean to prove linear independence of cotangent numbers without using the non-vanishing of Dirichlet's $L$ function $L(s, \chi)$ at unity, thus providing an elementary proof of infinitude of primes in an arithmetic progression. However, influenced by the spirit of Chowla's original 1970 [4] proof of linear independence of cotangent numbers using the non-vanishing of $L(1, \chi)$, numerous subsequent papers, except Baker-Birch-Wirsing [1], have been concerned with linear independence of allied cotangent numbers or what amounts to the same, non-vanishing of Maillet type determinants with negative order polylogarithm values, on the basis of non-vanishing of $L(1, \chi)$. Hence there must arise the expression of Maillet determinants in terms of $L(1, \chi)$ 's, which via the class number formula can be transformed into one involving relative class numbers. Thus at present what we understand by Chowla's problem is "the inverse problem of Chowla", i.e. one of proving linear independence of allied cotangent numbers by means of $L(1, \chi) \neq 0$.

2000 Mathematics Subject Classification: 11R42, 11C99. 
This has been most effectively treated by Funakura [9] by Maillet determinant method, but superseded in two different directions, one by Girstmair [10] using the character coordinates (matrix method) and the other by Milnor [25] using the notion of universality (module method), which seems inevitable more or less on the ground that the theory of determinants is just part of that of matrices. It is generally to be understood that the Maillet determinant method in Chowla's problem has been relegated into history, and that the interaction between them in the context of Girstmair [11] would be a plausible direction of further research.

However, from the point of view of the theory of Maillet determinants, Funakura's contribution is so fundamental that it already included some results by other authors published later (e.g. [5], see Part I).

In Part I, motivated by the work of Funakura and other fundamental ones by Endo [6-8] in conjunction with the work of Dohmae [5], Girstmair [12] and Hirabayashi [14], [15], we have introduced into the study of Maillet determinants a novel viewpoint of thinking of them as special values (or rather missing factors thereof) of the Dedekind zeta-function of an imaginary subfield of $\mathbb{Q}\left(\zeta_{m}\right), \zeta_{m}$ being the primitive $m$ th root of unity, at positive integral arguments.

While in Part I our main object of study was Maillet determinants of Bernoulli type, in this Part II we shall make a more essential use of polylogarithms in the spirit of Milnor [25], Yamamoto [27] and Lewin [20], [21] to consider Maillet determinants of Clausen type, as announced at the end of Part I.

As Corollary 2 below shows, Bernoulli type Maillet determinants alone do not suffice to express the special values of the Dedekind zeta-function, save for the rational and quadratic cases, and only when multiplied by Clausen type ones, they represent special values, hence the name "missing factors".

In the rational case, each type alone gives Euler's classical result on the values of the Riemann zeta-function.

Indeed, for even $k$ we deduce from Theorem 3 with $m=4, \chi=\chi_{0}^{*}$ $\left(\chi_{0}^{*}(n)=1\right.$ for all $\left.n \in \mathbb{N}\right)$ and $g=1$ that

$$
\zeta(k)=-\frac{(8 \pi i)^{k}}{4 \cdot k !\left(1-2^{k-1}\right)} B_{k}\left(\frac{1}{4}\right)=-\frac{(2 \pi i)^{k}}{2 \cdot k !} B_{k}(0), \quad \text { e.g. } \quad \zeta(2)=\frac{\pi^{2}}{6},
$$

while for odd $k \neq 1$,

$$
\zeta(k)=\frac{2(8 \pi i)^{k-1}}{k !\left(1-2^{k-1}\right)} A_{k}\left(\frac{1}{4}\right)=-\frac{(2 \pi i)^{k}}{k !} A_{k}(0),
$$

which by Lewin [21] simply reduces to Euler's classical representation (used by Apéry to prove the irrationality of $\zeta(3))$. 
We now turn to the quadratic field case. Viewing a given quadratic field as the maximal real subfield $K^{+}$of a suitable subfield $K$ of a certain cyclotomic field, we can evaluate the special values of the associated Dedekind zeta-function $\zeta_{K^{+}}(s)$.

The simplest case is where $K^{+}$is the maximal real subfield of a cyclotomic field $K$, and these occur only for $m=5,8,12$. For $m=5$, Corollary 2 to Theorem 3 gives

$$
\zeta_{\mathbb{Q}(\sqrt{5})}(2)=\frac{2 \pi^{4}}{75 \sqrt{5}}
$$

in conformity with the result of Zagier [29], while

$$
\zeta_{\mathbb{Q}(\sqrt{5})}(3)=-\frac{4 \pi^{2}}{3 \sqrt{5}} \zeta(3)\left\{A_{3}\left(\frac{1}{5}\right)-A_{3}\left(\frac{2}{5}\right)\right\},
$$

has some defects compared to the result of Zagier [29], in that (**) computes the values of a polylogarithm function at the arguments in the overfield $\mathbb{Q}\left(\zeta_{5}\right)$ (Zagier's result computes the same at arguments in the field $\mathbb{Q}(\sqrt{5})$ itself $)$.

As a slightly more complicated case, let $m=28, K=\mathbb{Q}\left(\zeta_{28}+\zeta_{28}^{9}+\zeta_{28}^{25}\right)$ $\subset \mathbb{Q}\left(\zeta_{28}\right)$ and $k=2,3$. Then $K^{+}=\mathbb{Q}(\sqrt{7})$ and

$$
\begin{gathered}
\zeta_{\mathbb{Q}(\sqrt{7})}(2)=\frac{\pi^{4}}{21 \sqrt{7}}, \\
\zeta_{\mathbb{Q}(\sqrt{7})}(3)=-\frac{2 \pi^{2}}{3 \sqrt{7}} \zeta(3)\left\{A_{3}\left(\frac{1}{28}\right)+\ldots-A_{3}\left(\frac{13}{28}\right)\right\} .
\end{gathered}
$$

Novel as they look, the formulas for quadratic fields are immediate consequences of the decomposition

$$
\zeta_{\mathbb{Q}(\sqrt{m})}(k)=\zeta(k) L\left(k,\left(\frac{*}{m}\right)\right),
$$

$\left(\frac{*}{m}\right)$ the Kronecker symbol, and Proposition 1 , as pointed out by the referee. E.g. for $m=5$, Proposition 1 gives

$$
L\left(3,\left(\frac{*}{5}\right)\right)=-\frac{4 \pi^{2}}{3 \sqrt{5}}\left(A_{3}\left(\frac{1}{5}\right)-A_{3}\left(\frac{2}{5}\right)\right),
$$

i.e. $(* *)$ above.

Apparently we can go on computing the values of $\zeta_{K^{+}}(s)\left(\right.$ or $\left.\zeta_{K}(s)\right)$ at other integral arguments, but we will not try to be exhaustive, leaving a more complete listing of evaluations to a forthcoming paper, in which we shall adopt an alternative simple approach using the partial or the Hurwitz zeta-function.

Hopefully, the values of Clausen functions are numerically computable with relative ease, and we can then obtain a numerical evaluation of zetavalues, which may result in some plausible conjectures on them. 
Acknowledgements. The authors would like to thank the referee for various important remarks and suggestions that led to the improvement of the paper. The authors also would like to thank Prof. R. Kučera for pointing out an error in our former paper [16] and for giving some enlightening examples.

2. A generalization of Bernoulli polynomials. Define the $k$ th ordinary Bernoulli polynomial $B_{k}(x)(k \geq 0)$ by the expansion

$$
F(t, x)=\frac{t e^{t x}}{e^{t}-1}=\sum_{k=0}^{\infty} B_{k}(x) \frac{t^{k}}{k !}
$$

$\left(B_{0}(x)=1, B_{1}(x)=x-1 / 2, B_{2}(x)=x^{2}-x+1 / 6, B_{3}(x)=x^{3}-3 x^{2} / 2+x / 2\right.$, etc.). Then $B_{k}=B_{k}(0)$ is the ordinary $k$ th Bernoulli number.

The $k$ th generalized Bernoulli polynomial $B_{k, \chi}(x)$, for a primitive Dirichlet character $\chi$ with conductor $f=f_{\chi}$, is similarly defined by

$$
F_{\chi}(t, x)=\sum_{a=1}^{f} \frac{\chi(a) t e^{(a+x) t}}{e^{f t}-1}=\sum_{k=0}^{\infty} B_{k, \chi}(x) \frac{t^{k}}{k !},
$$

and $B_{k, \chi}=B_{k, \chi}(0)$ is the $k$ th generalized Bernoulli number for $k \geq 0$, so that

$$
B_{k, \chi}(x)=f^{k-1} \sum_{a=0}^{f-1} \chi(a) B_{k}\left(\frac{x+a}{f}\right)
$$

and

$$
B_{k, \chi}=f^{k-1} \sum_{a=0}^{f-1} \chi(a) B_{k}\left(\frac{a}{f}\right)
$$

(cf. [26]).

The Bernoulli polynomials can be defined in a different way as the Fourier series, or through the $k$ th polylogarithm function $L i_{k}\left(e^{2 \pi i x}\right)$ with complex exponential argument (for $k \in \mathbb{N}, x \notin \mathbb{Z}$ )

$$
L i_{k}\left(e^{2 \pi i x}\right)=\sum_{n=1}^{\infty} e^{2 \pi i n x} n^{-k}=\sum_{n=1}^{\infty}(\cos 2 \pi n x+i \sin 2 \pi n x) n^{-k} .
$$

Coupled with Bernoulli polynomials $B_{k}(x)$ are Clausen functions $A_{k}(x)$, which we define through

$$
L i_{k}\left(e^{2 \pi i x}\right)=\frac{(2 \pi i)^{k-1}}{k !}\left\{A_{k}(x)-\pi i \bar{B}_{k}(x)\right\},
$$

where $\bar{B}_{k}(x)$ denotes the $k$ th periodic polynomial, i.e. the periodic function (with period 1) coinciding with $B_{k}(x)$ for $0 \leq x<1$. 
We define the value of $A_{k}(0)$ (the $k$ th Clausen number, so to say) on the ground of $(*)$ as

$$
A_{k}(0)= \begin{cases}\infty & \text { for } k=1, \\ 0 & \text { for } k \text { even } \\ \frac{k !}{(2 \pi i)^{k-1}} \zeta(k) & \text { for } k \text { odd }, \neq 1\end{cases}
$$

and define the $k$ th generalized Clausen number $A_{k, \chi}$ by

$$
\begin{aligned}
A_{k, \chi} & =f^{k-1} \sum_{a=0}^{f-1} \chi(a) A_{k}\left(\frac{a}{f}\right) \quad \text { for } \chi \neq \chi_{0}, \\
A_{k, \chi_{0}} & =A_{k}(0)
\end{aligned}
$$

where $\chi_{0}$ denotes the principal Dirichlet character to the relevant modulus.

From the definition, we have the following fundamental properties (cf. e.g. [19], [20], [26], [27]) $(k \in \mathbb{N})$.

Property 1 (Reciprocity law). For $0<x<1$,

$$
B_{k}(1-x)=(-1)^{k} B_{k}(x), \quad A_{k}(1-x)=(-1)^{k-1} A_{k}(x) .
$$

Property 2 (Distribution property). For any $N \in \mathbb{N}$,

$$
B_{k}(x)=N^{k-1} \sum_{a=0}^{N-1} B_{k}\left(\frac{x+a}{N}\right), \quad A_{k}(x)=N^{k-1} \sum_{a=0}^{N-1} A_{k}\left(\frac{x+a}{N}\right) .
$$

Property 3. Assume $\chi$ is a primitive Dirichlet character with conductor $f=f_{\chi}$ and $N$ is an integer such that $f \mid N$. Then

$$
B_{k, \chi}=N^{k-1} \sum_{a=0}^{N-1} \chi(a) B_{k}\left(\frac{a}{N}\right), \quad A_{k, \chi}=N^{k-1} \sum_{a=0}^{N-1} \chi(a) A_{k}\left(\frac{a}{N}\right)
$$

except for the case $k=1$ and $\chi=\chi_{0}$.

Moreover, let $\chi \neq \chi_{0}$ be a Dirichlet character mod $m$ with conductor $f=f_{\chi}$. Then for $k \in \mathbb{N}$,

$$
\begin{gathered}
m^{k-1} \sum_{a=0}^{m-1} \chi(a) B_{k}\left(\frac{a}{m}\right)=B_{k, \chi} \prod_{\substack{p \mid m \\
p \text { prime }}}\left(1-\chi^{*}(p) p^{k-1}\right), \\
m^{k-1} \sum_{a=0}^{m-1} \chi(a) A_{k}\left(\frac{a}{m}\right)=A_{k, \chi} \prod_{\substack{p \mid m \\
\text { p prime }}}\left(1-\chi^{*}(p) p^{k-1}\right)
\end{gathered}
$$

where $\chi^{*}$ is the primitive character corresponding to $\chi$.

From now on we make the convention that $B_{k, \chi}^{t}$ denotes $B_{k, \chi}$ for $t$ odd and $A_{k, \chi}$ for $t$ even. 
PROPERTY 4. The situation $B_{k, \chi}^{t}=0$ occurs only if $\chi$ is an even character when $k+t$ is odd, or $\chi$ is an odd character when $k+t$ is even, while in other cases $B_{k, \chi}^{t} \neq 0$ except for the case $k=1$ and $\chi=\chi_{0}$.

Let $L(s, \chi)$ be the Dirichlet $L$-series attached to a primitive Dirichlet character $\chi$ of conductor $f_{\chi}$, defined by

$$
L(s, \chi)=\sum_{n=1}^{\infty} \frac{\chi(n)}{n^{s}}=\prod_{p \text { prime }}\left(1-\chi(p) p^{-s}\right)^{-1} \quad \text { for } \Re(s)>1 .
$$

Proposition 1 (cf. [26], [27]). For $k \in \mathbb{N}$, if the primitive Dirichlet character $\chi$ with conductor $f=f_{\chi}$ satisfies $\chi(-1)=(-1)^{k}$, then

$$
L(k, \chi)=-\frac{(2 \pi i)^{k}}{2 k ! \tau(\bar{\chi})} \sum_{a=0}^{f-1} \bar{\chi}(a) B_{k}\left(\frac{a}{m}\right)=\left(\frac{2 \pi i}{f}\right)^{k-1} \frac{-\pi i}{k ! \tau(\bar{\chi})} B_{k, \bar{\chi}}
$$

where $\tau(\chi)=\sum_{a=1}^{f} \chi(a) e^{2 \pi i a / f}$ is the Gauss sum.

Moreover, if it satisfies $\chi(-1)=(-1)^{k-1}$ and $k \neq 1$, then

$$
L(k, \chi)=\frac{(2 \pi i)^{k-1}}{k ! \tau(\bar{\chi})} \sum_{a=0}^{f-1} \bar{\chi}(a) A_{k}\left(\frac{a}{m}\right)=\left(\frac{2 \pi i}{f}\right)^{k-1} \frac{1}{k ! \tau(\bar{\chi})} A_{k, \bar{\chi}} .
$$

3. Definitions and theorems. Let $m \geq 3$ be a fixed integer, let $G=$ $(\mathbb{Z} / m \mathbb{Z})^{*}$ and let $H$ be a subgroup of $G$ such that $-\overline{1} \notin H$. Let $X$ denote the set of all Dirichlet characters of $G$ trivial on $H$. For each integer $a$, let $R(a)$ denote the least positive residue modulo $m$ of $a$ defined by

$$
R(a) \equiv a \bmod m, \quad 0 \leq R(a)<m,
$$

and, in general, for each residue class $\bar{a}=a+m \mathbb{Z} \in G$, let $R(\bar{a})=R(a)$.

We write $\langle x\rangle$ for the fractional part of $x$, and $\langle x\rangle=x-[x]$, where [ ] denotes the Gauss symbol.

Definition 1. For $0 \leq x<1$, define

$$
\begin{array}{ll}
B_{k}^{0}(x)=B_{k}(x), & B_{k}^{1}(x)=A_{k}(x), \\
B_{k}^{2}(x)=B_{k}\left(\left\langle x+\frac{1}{2}\right\rangle\right), & B_{k}^{3}(x)=A_{k}\left(\left\langle x+\frac{1}{2}\right\rangle\right),
\end{array}
$$

and for each residue class $\bar{a}=a+m \mathbb{Z} \in G$, define the averaged Bernoulli polynomials

$$
\widetilde{B}_{k}^{t}\left(\frac{\bar{a}}{m}\right)=\sum_{\alpha \in a H} B_{k}^{t}\left(\frac{R(\alpha)}{m}\right)
$$

for $t=0,1,2,3$.

Definition 2 (A generalized Maillet determinant). Let $S \subset G$ denote a complete system of representatives of $G / H\{ \pm \overline{1}\}$ and let $2 g=|X|=|G / H|$. 
We define the shifted generalized Maillet determinant by

$$
D_{k}^{t}(x)=D_{k}^{t}[m, H, S](x)=\operatorname{det}\left(\widetilde{B}_{k}^{t}\left(\frac{\bar{a} \bar{b}^{-1}}{m}\right)+x\right)_{\bar{a}, \bar{b} \in S},
$$

for $k \in \mathbb{N}, t=0,1,2,3$ and $x \in \mathbb{R}$ except for $k=1, t=1$ and $k=1, t=3$ in which case

$$
D_{1}^{t}(x)=D_{1}^{t}[m, H, S](x)=\operatorname{det}\left(\widetilde{B}_{1}^{t}\left(\frac{\bar{a} \bar{b}^{-1}}{m}\right)-\widetilde{B}_{1}^{t}\left(\frac{\bar{a}}{m}\right)+x\right)_{\bar{a}, \bar{b} \in S} .
$$

In particular, we write

$$
D_{k}^{t}=D_{k}^{t}(0)
$$

for the generalized kth Maillet determinant.

TheOREM 1. For $m, G, H, S$ and $g$ as above, we have

$$
\begin{aligned}
D_{k}^{t}= & D_{k}^{t}[m, H, S](0) \\
= & \begin{cases}\left(\frac{m^{1-k}}{2}\right)^{g} \delta_{k}^{t} \prod_{\chi \in X^{k+1}} B_{k, \chi}^{t} & \text { for }(k, t) \neq(1,1),(1,3), \\
\left(\frac{1}{2}\right)^{g} \delta_{1}^{1} \prod_{\substack{\chi \in X^{2} \\
\chi \neq \chi_{0}}} A_{1, \chi} & \text { for }(k, t)=(1,1),(1,3),\end{cases}
\end{aligned}
$$

where $X^{k}$ is the subset of all even (resp. odd) characters of $X$ for $k$ even (resp. odd), $f=f_{\chi}$ the conductor of $\chi \in X, \chi^{*}$ is the primitive character corresponding to $\chi \in X$ and

$$
\begin{aligned}
& \delta_{k}^{0}=\prod_{\chi \in X^{k}} \delta_{k}(\chi),
\end{aligned}
$$

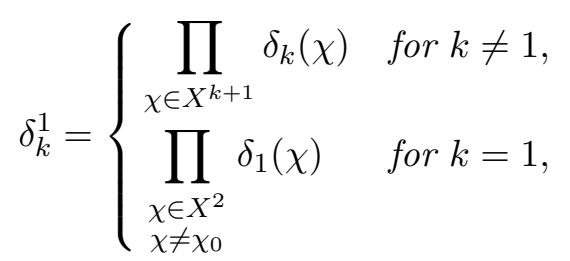

$$
\begin{aligned}
& \delta_{k}^{2}=\prod_{\chi \in X^{k}} \varepsilon_{k}(\chi),
\end{aligned}
$$

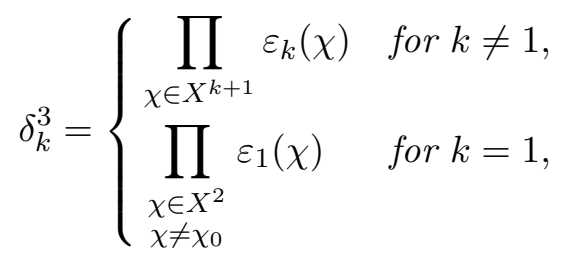


and where

$$
\begin{aligned}
& \delta_{k}(\chi)=\prod_{\substack{p \mid m \\
p \text { prime }}}\left(1-\chi^{*}(p) p^{k-1}\right), \\
& \varepsilon_{k}(\chi)=\left\{\begin{array}{l}
2^{1-k} \bar{\chi}^{*}(2) \prod_{\substack{p \mid 2 m \\
p \text { prime }}}\left(1-\chi^{*}(p) p^{k-1}\right) \quad \text { for } m \equiv 1 \bmod 2, \\
2^{k-1} \chi^{*}(2) \prod_{\substack{p \mid m / 2 \\
p \text { prime }}}\left(1-\chi^{*}(p) p^{k-1}\right) \quad \text { for } m \equiv 2 \bmod 4, \\
\prod_{\substack{p \mid m \\
p \text { prime }}}\left(1-\chi^{*}(p) p^{k-1}\right) \\
-\prod_{\substack{p \mid m \\
p \text { prime }}}\left(1-\chi^{*}(p) p^{k-1}\right) \quad \text { for } m \equiv 0 \bmod 4, m / f \text { even },
\end{array}\right.
\end{aligned}
$$

with $\chi^{*}$ denoting the primitive character corresponding to $\chi \in X$.

Theorem 2. Let $m, H, S, k$ and $t$ be as above. Then excepting the cases $k=1, t=1$ and $k=1, t=3$, we have

$$
D_{k}^{t}(x)=D_{k}^{t}[m, H, S](x)=\left(2 \Delta_{k}^{t} \cdot x+1\right) D_{k}^{t},
$$

provided that $\delta_{1}^{t} \neq 0$, where

$$
\Delta_{k}^{t}(a)= \begin{cases}m^{k-1} \sum_{\chi \in X^{k+t}} c_{\bar{\chi}} \chi(a) \delta_{k}(\chi)^{-1}\left(B_{k, \chi}\right)^{-1} & \text { for } t=1,2, \\ m^{k-1} \sum_{\chi \in X^{k+t}} c_{\bar{\chi}} \chi(a) \varepsilon_{k}(\chi)^{-1}\left(A_{k, \chi}\right)^{-1} & \text { for } t=3,4,\end{cases}
$$

and where

$$
\begin{aligned}
& c_{\chi}=c_{\chi}(S)=\sum_{\bar{a} \in S} \chi(R(\bar{a})), \\
& \Delta_{k}^{t}=\Delta_{k}^{t}[m, H, S]=\frac{1}{g} \sum_{\bar{a} \in S} \Delta_{k}^{t}(R(\bar{a})) .
\end{aligned}
$$

REMARK 1. Theorem 1 for $D_{k}^{2}$ gives a complete form of our former result on Endo's determinants ([16], Theorem 2(ii)) and for $D_{k}^{3}$ a complete analogue of the same. The non-vanishing condition of $\delta_{1}^{t}$ as well as the excepted cases $k=1, t=1,3$ are rather delicate. Therefore, we shall consider them later elsewhere (see however [12], [17]).

We shall also consider the problem of expressing $\delta_{k}^{t}$ in terms of quantities in $K$ in Theorem 2 elsewhere.

Now we turn to special applications of the results for $D_{k}^{0}$ and $D_{k}^{1}$. 
Theorem 3. Let $m, G, H, S$ be as in Theorem 1 . Then for $k \in \mathbb{N}$,

$$
\begin{aligned}
D_{k}^{0} & =\left(-\frac{k !}{(2 \pi i)^{k}}\right)^{g} \delta_{k}^{0} \prod_{\chi \in X^{k}}\left(\frac{f_{\chi}}{m}\right)^{k-1} \tau(\bar{\chi}) L(k, \chi), \\
D_{k}^{1} & =\left(\frac{k !}{2(2 \pi i)^{k-1}}\right)^{g} \delta_{k}^{1} \prod_{\chi \in X^{k+1}}\left(\frac{f_{\chi}}{m}\right)^{k-1} \tau(\bar{\chi}) L(k, \chi) \quad \text { for } k \neq 1, \\
D_{1}^{1} & =\left(\frac{1}{2}\right)^{g} \delta_{1}^{1} \prod_{\substack{\chi \in X^{2} \\
\chi \neq \chi_{0}}} \tau(\bar{\chi}) L(1, \chi),
\end{aligned}
$$

where $\tau(\chi)=\sum_{a=1}^{f_{\chi}} \chi(a) \zeta_{f_{\chi}}^{a}$ denotes the Gauss sum and $\bar{\chi}=\chi^{-1} \in X$. In particular, let $\mathbb{Q}\left(\zeta_{m}\right)$ be the mth cyclotomic field, $\zeta_{m}$ being a primitive mth root of unity and view $G$ as its Galois group $\operatorname{Gal}\left(\mathbb{Q}\left(\zeta_{m}\right) / \mathbb{Q}\right)$.

Further, let $K$ be the complex subfield of $\mathbb{Q}\left(\zeta_{m}\right)$ which corresponds to $H$. Then

$$
\begin{aligned}
D_{k}^{0} D_{k}^{1} & =\left(\frac{(-1)^{k} \pi(k !)^{2}}{(2 \pi)^{2 k}}\right)^{g} \delta_{k}^{0} \delta_{k}^{1} \prod_{\chi \in X}\left(\frac{f_{\chi}}{m}\right)^{k-1} \sqrt{|d(K)|} \cdot \zeta_{K}(k) \quad \text { for } k \neq 1, \\
D_{1}^{0} D_{1}^{1} & =\left(\frac{-\pi}{(2 \pi)^{2}}\right)^{g} \delta_{1}^{0} \delta_{1}^{1} \sqrt{|d(K)|} \prod_{\substack{\chi \in X \\
\chi \neq \chi_{0}}} L(1, \chi),
\end{aligned}
$$

where $d(K)$ is the discriminant of $K$ and $\zeta_{K}(s)$ is the Dedekind zeta-function of $K$.

REMARK 2. It was pointed out by Kučera [17] (as well as in a private communication on January 11,1999$)$ that our formula for $D_{S, 1}^{\prime}(0)\left(=D_{1}^{2}\right.$ in the present notation) in Theorem 1 of [16] is valid only for odd $m$ if we understand the meanings of constants $L(2)$ and $M(2)$ correctly, i.e. $L(2)=$ $L_{K}(2)=$ the number of primes in $K$ dividing 2 , and $M(2)=$ the inertia degree of 2 in $K$. Actually, our result for $D_{S, 1}^{\prime}(0)$ is correct up to

$$
D_{S, 1}^{\prime}(0)=\frac{h^{-}}{Q w} \delta_{1}^{2}
$$

where $\delta_{1}^{2}$ is defined in Theorem 1. Then the computation should have been done using the definition of $\delta_{1}^{2}$, and an argument in Remark after Theorem 1 in [16] is correct only for odd $m$. According to Kučera [17], the correct formula for $D_{S, 1}^{\prime}(0)$ should read

$$
D_{S, 1}^{\prime}(0)=\frac{h^{-}}{Q w} q_{K} z_{k}
$$

(for more details, see [16]). 
Corollary 1. We have

so that

$$
D_{1}^{t}= \begin{cases}\frac{(-1)^{g}}{Q w} \delta_{1}^{t} h^{-}(K) & \text { for } t=0,2 \\ \frac{(-1)^{g}}{2} \delta_{1}^{t} h\left(K^{+}\right) R_{K}^{+} & \text {for } t=1,3\end{cases}
$$

$$
D_{1}^{0} D_{1}^{1}=\frac{R_{K}}{2^{g} w} \delta_{1}^{0} \delta_{1}^{1} h(K),
$$

where $K^{+}$is the maximal real subfield of $K, R_{K}$ (resp. $R_{K^{+}}$) the regulator of $K\left(\right.$ resp. $\left.K^{+}\right), h(K)$ (resp. $\left.h\left(K^{+}\right)\right)$the class number of $K\left(\right.$ resp. $\left.K^{+}\right)$ and $h^{-}(K)=h(K) / h\left(K^{+}\right)$is the relative class number, and where $W$ is the group of roots of unity of $K, E$ (resp. $\left.E^{+}\right)$the unit group of $K$ (resp. $\left.K^{+}\right), w=|W|$ and $Q=\left[E: W E^{+}\right]$.

Proof. In Theorem 3 with $k=1$, we have

Now $R_{K} Q=2^{g-1} R_{K^{+}}$and

$$
\begin{aligned}
\prod_{\chi \in X^{1}} L(1, \chi) & =\frac{(2 \pi)^{g} h^{-}(K)}{Q w \sqrt{\left|\frac{d(K)}{d\left(K^{+}\right)}\right|}}, \\
\prod_{\chi \in X^{2}, \chi \neq \chi_{0}} L(1, \chi) & =\frac{2^{g} h\left(K^{+}\right) R_{K^{+}}}{2 \sqrt{\left|d\left(K^{+}\right)\right|}} .
\end{aligned}
$$

$$
\prod_{\chi \in X^{1}} \tau(\chi)=i^{g} \sqrt{\left|\frac{d(K)}{d\left(K^{+}\right)}\right|}
$$

prove the equalities.

Corollary 2. Let $m=5, K=\mathbb{Q}\left(\zeta_{5}\right)$ and $k=2,3$. Then $K^{+}=\mathbb{Q}(\sqrt{5})$, and

$$
\begin{aligned}
\zeta_{\mathbb{Q}\left(\zeta_{5}\right)}(2) & =\frac{8 \pi^{6}}{375 \sqrt{5}}\left\{A_{2}\left(\frac{1}{5}\right)^{2}-A_{2}\left(\frac{2}{5}\right)^{2}\right\} \\
\zeta_{\mathbb{Q}\left(\zeta_{5}\right)}(3) & =-\frac{64 \pi^{8}}{5^{7 \frac{1}{2}}} \zeta(3)\left\{A_{3}\left(\frac{1}{5}\right)-A_{3}\left(\frac{2}{5}\right)\right\} \\
\zeta_{\mathbb{Q}(\sqrt{5})}(2) & =\frac{2 \pi^{4}}{75 \sqrt{5}}, \\
\zeta_{\mathbb{Q}(\sqrt{5})}(3) & =-\frac{4 \pi^{2}}{3 \sqrt{5}} \zeta(3)\left\{A_{3}\left(\frac{1}{5}\right)-A_{3}\left(\frac{2}{5}\right)\right\} .
\end{aligned}
$$

Proof. We know that (data 1)

$$
\begin{aligned}
g & =2 \\
\delta_{k}\left(\chi_{0}\right) & =1-5^{k-1}, \\
\delta_{k}(\chi) & =1 \quad \text { for } \chi \neq \chi_{0} \\
\sqrt{|d(K)|} & =5 \sqrt{5} .
\end{aligned}
$$


By Theorem 3,

(data 2)

$$
\begin{aligned}
D_{2}^{0} D_{2}^{1} & =-\frac{1}{16 \pi^{6}}(1-5) \frac{1}{5}(-5 \sqrt{5}) \zeta_{K}(2) \\
D_{3}^{0} D_{3}^{1} & =-\frac{81}{256 \pi^{10}}\left(1-5^{2}\right)\left(\frac{1}{5}\right)^{2}(-5 \sqrt{5}) \zeta_{K}(3) \\
D_{2}^{0} & =\frac{1}{4 \pi^{4}}(1-5) \frac{1}{5} \cdot \sqrt{5} \zeta_{K^{+}}(2) \\
D_{3}^{1} & =\frac{9}{16 \pi^{4}}\left(1-5^{2}\right)\left(\frac{1}{5}\right)^{2} \sqrt{5} \zeta_{K^{+}}(3)
\end{aligned}
$$

From Proposition 2,

(data 3)

$$
\begin{aligned}
A_{3}\left(\frac{1}{5}\right)+A_{3}\left(\frac{2}{5}\right) & =\frac{1}{2}\left(5^{-2}-1\right) A_{3}(0) \\
& =-\frac{12}{25} \cdot \frac{3 !}{(2 \pi i)^{2}} \zeta(3) .
\end{aligned}
$$

Using these, we can derive the desired equalities.

The proof of the following corollaries being the same, we just state the data necessary for the proof.

Corollary 3. Let $m=12, K=\mathbb{Q}\left(\zeta_{12}\right)$ and $k=2,3$. Then $K^{+}=$ $\mathbb{Q}(\sqrt{3})$, and

$$
\begin{aligned}
& \zeta_{\mathbb{Q}\left(\zeta_{12}\right)}(2)=\frac{\pi^{6}}{9}\left\{A_{2}\left(\frac{1}{12}\right)^{2}-A_{2}\left(\frac{5}{12}\right)^{2}\right\} \\
& \zeta_{\mathbb{Q}\left(\zeta_{12}\right)}(3)=-\frac{25 \pi^{8}}{2^{6} \cdot 3^{8}} \zeta(3)\left\{A_{3}\left(\frac{1}{12}\right)-A_{3}\left(\frac{5}{12}\right)\right\} .
\end{aligned}
$$

Proof.

(data 1)

$$
\begin{aligned}
g & =2, \\
\delta_{k}\left(\chi_{0}\right) & =\left(1-2^{k-1}\right)\left(1-3^{k-1}\right), \\
\delta_{k}(\chi) & =1 \quad \text { for } \chi \neq \chi_{0}, \\
\sqrt{|d(K)|} & =12,
\end{aligned}
$$

(data 2$) \quad D_{2}^{0} D_{2}^{1}=-\frac{1}{16 \pi^{6}}(1-2)(1-3) \frac{1}{12} \cdot \frac{6}{12}(-12) \zeta_{K}(2)$,

$$
D_{3}^{0} D_{3}^{1}=-\frac{81}{32 \pi^{10}}\left(1-2^{2}\right)\left(1-3^{2}\right)\left(\frac{1}{12}\right)^{2} \cdot\left(\frac{5}{12}\right)^{2}(-12) \zeta_{K}(3)
$$

(data 3)

$$
\begin{aligned}
A_{3}\left(\frac{1}{12}\right)+A_{3}\left(\frac{5}{12}\right) & =\frac{\left(1-2^{2}\right)\left(1-3^{2}\right)}{2 \cdot 12^{2}} A_{3}(0) \\
& =\frac{1}{12} \cdot \frac{3 !}{(2 \pi i)^{2}} \zeta(3) .
\end{aligned}
$$


Corollary 4. Let $m=8, K=\mathbb{Q}\left(\zeta_{8}\right)$ and $k=2,3$. Then $K^{+}=$ $\mathbb{Q}(\sqrt{2})$, and

$$
\begin{aligned}
& \zeta_{\mathbb{Q}\left(\zeta_{8}\right)}(2)=\frac{\pi^{6}}{24}\left\{A_{2}\left(\frac{1}{8}\right)^{2}-A_{2}\left(\frac{3}{8}\right)^{2}\right\}, \\
& \zeta_{\mathbb{Q}\left(\zeta_{8}\right)}(3)=-\frac{\pi^{8}}{2^{14}} \zeta(3)\left\{A_{3}\left(\frac{1}{8}\right)-A_{3}\left(\frac{3}{8}\right)\right\} .
\end{aligned}
$$

Proof.

(data 1)

$$
\begin{aligned}
g & =2, \\
\delta_{k}\left(\chi_{0}\right) & =1-2^{k-1}, \\
\delta_{k}(\chi) & =1 \quad \text { for } \chi \neq \chi_{0}, \\
\sqrt{|d(K)|} & =16,
\end{aligned}
$$

(data 2$) \quad D_{2}^{0} D_{2}^{1}=-\frac{1}{16 \pi^{6}}(1-2) \frac{1}{8} \cdot \frac{4}{8}(-16) \zeta_{K}(2)$,

$$
D_{3}^{0} D_{3}^{1}=-\frac{81}{256 \pi^{10}}\left(1-2^{2}\right)\left(\frac{1}{8}\right)^{2} \cdot\left(\frac{4}{8}\right)^{2}(-16) \zeta_{K}(3),
$$

(data 3$)$

$$
\begin{aligned}
A_{3}\left(\frac{1}{8}\right)+A_{3}\left(\frac{3}{8}\right) & =\frac{1-2^{2}}{2 \cdot 8^{2}} A_{3}(0) \\
& =-\frac{3}{128} \cdot \frac{3 !}{(2 \pi i)^{2}} \zeta(3) .
\end{aligned}
$$

We can also generalize the result of Mestre-Schappacher ([23], 3.4).

Corollary 5. Let $m, K, S$ and $g$ be as in Theorem 3 . Then for $k \in 2 \mathbb{N}$,

$$
D_{k}^{0}=\left(-\frac{k}{2 m^{k-1}}\right)^{g} \delta_{k}^{0} \prod_{\chi \in X^{k}} L(1-k, \chi)=\left(-\frac{k}{2 m^{k-1}}\right)^{g} \delta_{k}^{0} \zeta_{K^{+}}(1-k),
$$

and for $k \in 2 \mathbb{N}+1, \zeta_{K^{+}}(1-k)=0$.

Proof. We know that $L(1-k, \chi)=-B_{k, \chi} / k$ for $k \in \mathbb{N}$ (cf. [26], Thm. 4.2). Using this and Theorem 1 , we can derive the desired equalities.

\section{Proof of theorems}

Lemma 1 (Dedekind-Frobenius determinant, cf. [18], [26]). Let $G$ be a finite abelian group, let $N$ be a subgroup of $G$ and let $T \subset G$ be a complete system of representatives of $G / N$. For a character $\lambda$ of $N$, let $\Delta$ be the set of all characters of $G$ whose restriction to $N$ is $\lambda$. Then for any complex-valued function $f$ on $G$ with

$$
f(a h)=\lambda(h) f(a) \quad(a \in G, h \in N),
$$


we have

$$
\operatorname{det}\left(f\left(a b^{-1}\right)\right)_{a, b \in T}=\prod_{\chi \in \Delta}\left(\sum_{a \in T} \bar{\chi}(a) f(a)\right)
$$

and if $\chi_{0} \in \Delta$ then

$$
\operatorname{det}\left(f\left(a b^{-1}\right)-f(a)\right)_{\substack{a, b \in T \\ a, b \notin N}}=\prod_{\substack{\chi \in \Delta \\ \chi \neq \chi_{0}}}\left(\sum_{\substack{a \in T \\ a \notin N}} \bar{\chi}(a) f(a)\right) .
$$

Proof of Theorem 1. We apply Lemma 1 with $G=(\mathbb{Z} / m \mathbb{Z})^{*}, N=$ $H\{ \pm \overline{1}\}, T=S, \Delta=X^{k+t}$ and $f(\bar{a})=\widetilde{B}_{k}^{t}(\bar{a} / m)$. We know that $\lambda \in X^{k+t}$ satisfies $\left.\lambda\right|_{H}=1$ and $\lambda(-1)=(-1)^{k+t}$. We treat each case of $t$ separately.

The case $t=0$. Mostly from the definitions of the quantities involved (with Property 3 applied at the last but one stage) we infer that

$$
\begin{aligned}
D_{k}^{0}(0) & =\operatorname{det}\left(\widetilde{B}_{k}^{0}\left(\frac{\bar{a} \bar{b}^{-1}}{m}\right)\right)_{\bar{a}, \bar{b} \in S}=\prod_{\chi \in X^{k}} \sum_{\bar{a} \in S} \chi(\bar{a}) \widetilde{B}_{k}\left(\frac{\bar{a}}{m}\right) \\
& =\prod_{\chi \in X^{k}} \sum_{\bar{a} \in S} \sum_{\alpha \in \bar{a} H} \chi(\alpha) B_{k}\left(\frac{R(\alpha)}{m}\right) \\
& =\prod_{\chi \in X^{k}} \frac{1}{2} \sum_{a=0}^{m-1} \chi(a) B_{k}\left(\frac{a}{m}\right) \\
& =\prod_{\chi \in X^{k}} \frac{m^{1-k}}{2} B_{k, \chi} \prod_{\substack{p \mid m \\
p \text { prime }}}\left(1-\chi^{*}(p) p^{k-1}\right) \\
& =\left(\frac{m^{1-k}}{2}\right)^{g} \sum_{k}^{0} \prod_{\chi \in X^{k}} B_{k, \chi} .
\end{aligned}
$$

The case $t=1$. Similarly to the case $t=0$, for $k \neq 1$ we have

$$
\begin{aligned}
D_{k}^{1}(0) & =\operatorname{det}\left(\widetilde{B}_{k}^{1}\left(\frac{\bar{a} \bar{b}^{-1}}{m}\right)\right)_{\bar{a}, \bar{b} \in S}=\prod_{\chi \in X^{k+1}} \sum_{\bar{a} \in S} \chi(\bar{a}) \widetilde{A}_{k}\left(\frac{\bar{a}}{m}\right) \\
& =\prod_{\chi \in X^{k+1}} \frac{1}{2} \sum_{a=0}^{m-1} \chi(a) A_{k}\left(\frac{a}{m}\right)=\left(\frac{m^{1-k}}{2}\right)^{g} \delta_{k}^{1} \prod_{\chi \in X^{k+1}} A_{k, \chi}
\end{aligned}
$$

and

$$
D_{1}^{1}(0)=\left(\frac{1}{2}\right)^{g-1} \delta_{1}^{1} \prod_{\substack{\chi \in X^{2} \\ \chi \neq \chi_{0}}} A_{1, \chi}
$$


The case $t=2$. It suffices to transform the sum

$$
S_{k, \chi}=\sum_{a=0}^{m-1} \chi(a) B_{k}\left(\left\langle\frac{a}{m}+\frac{1}{2}\right\rangle\right)
$$

to an appropriate form. Note that by Property 2, for each integer $a$ with $0 \leq a<m$ we have

$$
B_{k}\left(\left\langle\frac{a}{m}+\frac{1}{2}\right\rangle\right)=-B_{k}\left(\frac{a}{m}\right)+2^{1-k} B_{k}\left(\left\langle\frac{2 a}{m}\right\rangle\right) .
$$

We distinguish four cases.

First, if $m \equiv 1 \bmod 2$, then

$$
\begin{aligned}
S_{k, \chi} & =\sum_{a=0}^{m-1}\left\{-\chi(a) B_{k}\left(\frac{a}{m}\right)+2^{1-k} \chi^{-1}(2) \chi(2 a) B_{k}\left(\left\langle\frac{2 a}{m}\right\rangle\right)\right\} \\
& =-\left(1-2^{1-k} \chi^{-1}(2)\right) \sum_{a=0}^{m-1} \chi(a) B_{k}\left(\frac{a}{m}\right) \\
& =\varepsilon_{k}(\chi) m^{1-k} B_{k, \chi} .
\end{aligned}
$$

Secondly, if $m \equiv 2 \bmod 4$, there exists a Dirichlet character $\xi \bmod m / 2$ whose primitive character $\xi^{*}$ is equal to that of $\chi$, and $\xi(\bar{a})=\chi(\bar{a})$ for each $\bar{a} \in G$. Thus

$$
\begin{aligned}
S_{k, \chi} & =-\sum_{a=0}^{m-1} \chi(a) B_{k}\left(\frac{a}{m}\right)+2^{1-k} \sum_{\substack{0 \leq a<m \\
a \text { odd }}} \xi(a) B_{k}\left(\left\langle\frac{a}{m / 2}\right\rangle\right) \\
& =-\sum_{a=0}^{m-1} \chi(a) B_{k}\left(\frac{a}{m}\right)+2^{1-k} \sum_{a=0}^{m / 2-1} \xi(a) B_{k}\left(\frac{a}{m / 2}\right) \\
& =-\delta_{k}(\chi) m^{1-k} B_{k, \chi}+2^{1-k} \delta_{k}(\xi)\left(\frac{m}{2}\right)^{1-k} B_{k, \xi} .
\end{aligned}
$$

By the definition of $\xi$, we have $B_{k, \xi}=B_{k, \chi}$ and

$$
\delta_{k}(\chi)=\delta_{k}(\xi)\left(1-\chi^{*}(2) 2^{k-1}\right) .
$$

Hence, when $\delta_{k}(\chi) \neq 0$,

$$
\begin{aligned}
S_{k, \chi} & =\delta_{k}(\chi) m^{1-k}\left(-1+\left(1-2^{k-1} \chi^{*}(2)\right)^{-1}\right) B_{k, \chi} \\
& =-\left(1-2^{1-k} \bar{\chi}^{*}(2)\right)^{-1} \delta_{k}(\chi) m^{1-k} B_{k, \chi} \\
& =\varepsilon_{k}(\chi) m^{1-k} B_{k, \chi} .
\end{aligned}
$$

Thirdly, if $m \equiv 0 \bmod 4$ and $m$ is divisible by $2 f_{\chi}$, then, as in the second case, there exists a Dirichlet character $\xi \bmod m / 2$ whose primitive character $\xi^{*}$ is equal to that of $\chi, \xi(\bar{a})=\chi(\bar{a})$ for each $\bar{a} \in G$. In this case $B_{k, \xi}=B_{k, \chi}$ 
and $\delta_{k}(\xi)=\delta_{k}(\chi)$. Thus

$$
\begin{aligned}
S_{k, \chi} & =-\sum_{a=0}^{m-1} \chi(a) B_{k}\left(\frac{a}{m}\right)+2^{1-k} \sum_{a=0}^{m-1} \xi(a) B_{k}\left(\left\langle\frac{a}{m / 2}\right\rangle\right) \\
& =-\sum_{a=0}^{m-1} \chi(a) B_{k}\left(\frac{a}{m}\right)+2^{1-k} \cdot 2 \sum_{a=0}^{m / 2-1} \xi(a) B_{k}\left(\frac{a}{m / 2}\right) \\
& =-\delta_{k}(\chi) m^{1-k} B_{k, \chi}+2^{1-k} \cdot 2 \delta_{k}(\xi)\left(\frac{m}{2}\right)^{1-k} B_{k, \xi} \\
& =\delta_{k}(\chi) m^{1-k} B_{k, \chi} .
\end{aligned}
$$

Lastly, if $m \equiv 0 \bmod 4$ and $m$ is not divisible by $2 f_{\chi}$, then $\chi(m / 2+1)=$ -1 . So

$$
\begin{aligned}
\chi\left(\frac{m}{2}+a\right) B_{k}\left(\left\langle\frac{2(m / 2+a)}{m}\right\rangle\right) & =\chi\left(\frac{m}{2}+1\right) \chi(a) B_{k}\left(\left\langle\frac{2 a}{m}\right\rangle\right) \\
& =-\chi(a) B_{k}\left(\left\langle\frac{2 a}{m}\right\rangle\right) .
\end{aligned}
$$

Hence

$$
S_{k, \chi}=-\sum_{a=0}^{m-1} \chi(a) B_{k}\left(\frac{a}{m}\right)=-\delta_{k}(\chi) m^{1-k} B_{k, \chi} .
$$

Putting all together, we conclude in the case $t=2$ that

$$
\begin{aligned}
D_{k}^{2}(0) & =\operatorname{det}\left(\widetilde{B}_{k}^{2}\left(\frac{\bar{a} \bar{b}^{-1}}{m}\right)\right)_{\bar{a}, \bar{b} \in S}=\prod_{\chi \in X^{k+2}} \frac{1}{2} S_{k, \chi} \\
& =\left(\frac{m^{1-k}}{2}\right)^{g} \delta_{k}^{2} \prod_{\chi \in X^{k+2}} B_{k, \chi} .
\end{aligned}
$$

The case $t=3$ being reduced to other cases, this completes the proof.

Proof of Theorem 2. We need to evaluate the sum

$$
\sum_{\bar{a} \in S} \Delta_{k}^{t}(R(\bar{a})) \widetilde{B}_{k}^{t}\left(\frac{\bar{a} \bar{b}^{-1}}{m}\right)
$$

First note that by definition, Property 3 and Property 4,

$$
\Delta_{k}^{t}(a)=\sum_{\chi \in X^{k+t}} c_{\bar{\chi}} \chi(a)\left\{\sum_{b=0}^{m-1} \chi(b) B_{k}^{t}\left(\frac{b}{m}\right)\right\}^{-1} .
$$


Next, by orthogonality property of characters of $G / H$,

$$
\begin{aligned}
\widetilde{B}_{k}^{t}\left(\frac{\bar{a} \bar{b}^{-1}}{m}\right) & =\sum_{\bar{\alpha} \in \bar{a} \bar{b}^{-1} H} B_{k}^{t}\left(\frac{R(\bar{\alpha})}{m}\right) \\
& =\frac{1}{\varphi(m)} \sum_{\bar{\alpha} \in \bar{a} \bar{b}^{-1} H} \sum_{\chi \in X} \bar{\chi}(R(\bar{\alpha})) \sum_{c=0}^{m-1} B_{k}^{t}\left(\frac{c}{m}\right) \chi(c) \\
& =\frac{1}{\varphi(m)} \cdot \frac{\varphi(m)}{2 g} \sum_{\chi \in X^{k+t}} \bar{\chi}\left(R\left(\bar{a} \bar{b}^{-1}\right)\right) \sum_{c=0}^{m-1} \chi(c) B_{k}^{t}\left(\frac{c}{m}\right),
\end{aligned}
$$

where in the last step we used Property 4.

Hence the sum in question becomes

$$
\begin{aligned}
\frac{1}{2 g} \sum_{\bar{a} \in S} \sum_{\psi, \chi \in X^{k+t}} c_{\bar{\psi}} \psi(R(\bar{a}))\left\{\sum_{d=0}^{m-1} \psi(d) B_{k}^{t}\left(\frac{d}{m}\right)\right\}^{-1} & \\
& \times \bar{\chi}\left(R\left(\bar{a} \bar{b}^{-1}\right)\right) \sum_{c=0}^{m-1} \chi(c) B_{k}^{t}\left(\frac{c}{m}\right) \\
= & \frac{1}{2 g} \sum_{\chi, \psi \in X^{k+t}} c_{\bar{\psi}} \chi(R(\bar{b}))\left\{\sum_{d=0}^{m-1} \psi(d) B_{k}^{t}\left(\frac{d}{m}\right)\right\}^{-1} \\
& \times \sum_{c=0}^{m-1} \chi(c) B_{k}^{t}\left(\frac{c}{m}\right) \sum_{\bar{a} \in S}(\psi \bar{\chi})(R(\bar{a})) \\
= & \frac{1}{2} \sum_{\chi \in X^{k+t}} c_{\bar{\chi}} \chi(R(\bar{b})),
\end{aligned}
$$

by orthogonality of characters.

Recalling the definition of $c_{\chi}$ in Theorem 2, we see that

$$
\sum_{\bar{a} \in S} \Delta_{k}^{t}(R(\bar{a})) \widetilde{B}_{k}^{t}\left(\frac{\bar{a} \bar{b}^{-1}}{m}\right)=\frac{1}{2} \sum_{\bar{a} \in S} \sum_{\chi \in X^{k+t}} \bar{\chi}(R(\bar{a})) \chi(R(\bar{b}))=\frac{1}{2} g
$$

by orthogonality of even characters of $X$.

Hence, in particular, there is an $\bar{a}_{0} \in S$ such that $\Delta_{k}^{t}\left(R\left(\bar{a}_{0}\right)\right) \neq 0$.

We define the matrix $M_{k}^{t}(x)$ as follows:

$$
\begin{aligned}
M_{k}^{t}(x) & =\left(m_{k, \bar{a} \bar{b}}^{t}\right)_{\bar{a}, \bar{b} \in S}, \\
m_{k, \bar{a} \bar{b}}^{t} & = \begin{cases}\widetilde{B}_{k}^{t}\left(\frac{\bar{a}^{-1}}{m}\right)+x, & \bar{a} \neq \bar{a}_{0}, \\
1, & \bar{a}=\bar{a}_{0} .\end{cases}
\end{aligned}
$$


We easily see that $\operatorname{det} M_{k}^{t}(x)=\operatorname{det} M_{k}^{t}(0)$. For the matrix $M$, we denote the $(i, j)$-cofactor by $M_{i, j}$.

Expanding $D_{k}^{t}(x)$ with respect to the $a_{0}$ th row, we have

$$
\begin{aligned}
\Delta_{k}^{t}\left(R\left(\bar{a}_{0}\right)\right) D_{k}^{t}(x) & =\Delta_{k}^{t}\left(R\left(\bar{a}_{0}\right)\right) \sum_{\bar{b} \in S}\left\{\widetilde{B}_{k}^{t}\left(\frac{\bar{a}_{0} \bar{b}^{-1}}{m}\right)+x\right\} M_{k}^{t}(x)_{\bar{a}_{0}, \bar{b}} \\
& =\sum_{\bar{a} \in S} \Delta_{k}^{t}(R(\bar{a})) \sum_{\bar{b} \in S}\left\{\widetilde{B}_{k}^{t}\left(\frac{\bar{a} \bar{b}^{-1}}{m}\right)+x\right\} M_{k}^{t}(x)_{\bar{a}_{0}, \bar{b}} .
\end{aligned}
$$

Since for $\bar{a} \neq \bar{a}_{0}$, the inner sum is 0 , substituting the above expression and noting that $\sum_{\bar{a} \in S} \Delta_{k}^{t}(R(\bar{a})) x=g \Delta_{k}^{t} x$, we conclude that

$$
\Delta_{k}^{t}\left(R\left(\bar{a}_{0}\right)\right) D_{k}^{t}(x)=g\left(\Delta_{k}^{t} x+\frac{1}{2}\right) \sum_{\bar{b} \in S} M_{k}^{t}(x)_{\bar{a}_{0}, \bar{b}}=g\left(\Delta_{k}^{t} x+\frac{1}{2}\right) \operatorname{det} M_{k}^{t}(x) .
$$

Comparing this with the special case with $x=0$, we get

$$
\Delta_{k}^{t}\left(R\left(\bar{a}_{0}\right)\right) D_{k}^{t}(x)=\Delta_{k}^{t}\left(R\left(\bar{a}_{0}\right)\right)\left(2 \Delta_{k}^{t} x+1\right) D_{k}^{t}(0),
$$

whence the assertion follows.

Proof of Theorem 3. By Theorem 1, Proposition 1 and $\prod_{\chi \in X} \tau(\chi)=$ $i^{g} \sqrt{|d(K)|}$, the result follows.

REMARK 3. Now that we have two extensive lists of references on Maillet determinants and Demyanenko matrices, namely, one in Part I and the other due to Yamamura [28], we shall list up only those references which are (i) directly connected with the content of the present paper, (ii) missing in both lists above, (iii) so informative that they can be used as source files to trace back previous works.

Some of them are worth being paid special attention.

Bundschuh's paper [2] is concerned with an extension of Chowla's problem to the case of derivatives of the cotangent function, or the Clausen functions of negative order, giving a new proof of the main result of Okada and of Wang. Okada's and Wang's result was, however, properly criticised by Girstmair [10] on the ground that it follows from a very simple observation on an infinite series. However, from the point of view of Maillet determinants, Bundschuh's Lemma 5 and formula (9) give

$$
\operatorname{det}\left(\frac{k !}{(-2 \pi i)^{k}} A_{1-k}\left(\frac{a b^{-1}}{q}\right)\right)=\prod_{\substack{\chi \in \widehat{\mathbb{Z}}_{q}^{*} \\ \chi(-1)=(-1)^{k}}}\left(-\frac{k !}{(2 \pi i)^{k}} q^{k} L(k, \bar{\chi})\right) .
$$

Bundschuh proceeds further to give quantitative versions of these types of theorems. 
Metsänkylä's letter to the editor [24] is worthy of attention as it gives a detailed information on the estimate of the relative class number of a special type of cyclotomic fields.

We have intentionally avoided the references on Demyanenko matrix, except for those which are closely related to Maillet determinants, considering them out of scope. Now, Hirabayashi [15] has succeeded for the first time in unifying these two notions in an essential way using the idea of Girstmair.

REMARK 4. Hazama's recent paper [13] has come into our attention, which calculates the special values of the Dedekind zeta-functions of prime cyclotomic fields. The essential ingredient is the function $f_{n}(a)$, which is nothing but

$$
\frac{1}{n}(2 m)^{n-1}\left(B_{n}\left(\left\langle\frac{a}{m}+\frac{1}{2}\right\rangle\right)-B_{n}\left(\frac{a}{m}\right)\right) .
$$

Hence our argument in the proof of Theorem 1, valid for composite $m$, applies to this situation as well, to produce similar results in terms of Demyanenko determinants.

We shall consider this setting soon elsewhere.

\section{References}

[1] A. Baker, B. J. Birch and E. Wirsing, On a problem of Chowla, J. Number Theory 5 (1973), 224-236.

[2] P. Bundschuh, On the linear independence of certain algebraic numbers, Keio Sem. Math. Sci. 12 (1988), 11-30.

[3] L. Carlitz and F. R. Olson, Maillet's determinant, Proc. Amer. Math. Soc. 6 (1955), 265-269.

[4] S. Chowla, The nonexistence of nontrivial linear relations between the roots of a certain irreducible equation, J. Number Theory 2 (1970), 120-123.

[5] K. Dohmae, Demjanenko matrix for imaginary abelian fields of odd conductors, Proc. Japan Acad. Ser. A 70 (1994), 292-294.

[6] A. Endo, The relative class number of certain imaginary abelian fields, Abh. Math. Sem. Univ. Hamburg 58 (1988), 237-243.

[7] - , The relative class numbers of certain imaginary abelian number fields and determinants, J. Number Theory 34 (1990), 13-20.

[8] - On the Stickelberger ideal of $(2, \ldots, 2)$-extensions of a cyclotomic number field, Manuscripta Math. 69 (1990), 107-132.

[9] T. Funakura, On Kronecker's limit formula for Dirichlet series with periodic coefficients, Acta Arith. 55 (1990), 59-73.

[10] K. Girstmair, Character coordinates and annihilators of cyclotomic numbers, Manuscripta Math. 59 (1987), 375-389.

[11] —, An index formula for the relative class number of an abelian field, J. Number Theory 32 (1989), 100-110.

[12] - The relative class numbers of imaginary cyclic fields of degrees 4, 6, 8, and 10, Math. Comp. 61 (1993), 881-887, S25-S27. 
[13] F. Hazama, Determinantal formula for the cuspidal class number of the modular curve $X_{1}(m)$, J. Number Theory 68 (1998), 229-242.

[14] M. Hirabayashi, A relative class number formula for an imaginary abelian field by means of Demjanenko matrix, in: Proc. Conf. on Analytic and Elementary Number Theory (Vienna, 1996), W. G. Nowak and J. Schoißengeier (eds.), Universität Wien and Universität für Bodenkultur, Wien, 1997, 81-91.

[15] —, A generalization of Maillet and Demyanenko determinants, Acta Arith. 83 (1998), 391-397.

[16] S. Kanemitsu and T. Kuzumaki, On generalization of the Maillet determinant, in: Proc. Number Theory Conf. (Eger, 1997), de Gruyter, 1998, 271-287.

[17] R. Kučera, Formulae for the relative class number of an imaginary abelian field in the form of a determinant, preprint.

[18] S. Lang, Cyclotomic Fields, Grad. Texts in Math. 59, Springer, 1978.

[19] D. H. Lehmer, A new approach to Bernoulli polynomials, Amer. Math. Monthly 95 (1988), 905-911.

[20] L. Lewin, Polylogarithms and Associated Functions, North-Holland, 1981.

[21] —, Structural Properties of Polylogarithms, Math. Surveys Monographs 37, Amer. Math. Soc., Providence, RI, 1991.

[22] E. Maillet, Quest. 4269, L'Intermédiaire des Math. 20 (1913), 218, reply by E. Malo, ibid. 21 (1914), 173-176.

[23] J.-F. Mestre et N. Schappacher, Séries de Kronecker et fonctions L des puissances symétriques de courbes elliptiques sur $\mathbb{Q}$, in: Arithmetic Algebraic Geometry, G. van der Geer et al. (eds.), Progr. Math. 89, Birkhäuser, 1991, 209-245.

[24] T. Metsänkylä, Letter to the editor, J. Number Theory 64 (1997), 162-163.

[25] J. Milnor, On polylogarithms, Hurwitz zeta functions, and the Kubert identities, Enseign. Math. (2) 29 (1983), 281-322.

[26] L. Washington, Introduction to Cyclotomic Fields, Grad. Texts in Math. 83, Springer, 1983.

[27] Y. Yamamoto, Dirichlet series with periodic coefficients, in: Algebraic Number Theory (Kyoto, 1976), Japan Soc. Promotion Sci., 1977, 275-289.

[28] K. Yamamura, The list of papers concerning the relation between the determinants and the absolute class number of the imaginary abelian field, unpublished (in Japanese).

[29] D. Zagier, Values of zeta function and their applications, in: First European Congr. of Math. (Paris, 1992), A. Joseph et al. (eds.), Progr. Math. 120, Vol. II: Invited Lectures (Part 2), Birkhäuser, 1994, 497-512.

Graduate School of

Advanced Technology

University of Kinki

Iizuka, Fukuoka 820-8555, Japan

E-mail: kanemitu@fuk.kindai.ac.jp
Department of Electrical Engineering Faculty of Engineering Gifu University Gifu 501-1193, Japan E-mail: kuzumaki@cc.gifu-u.ac.jp

Received on 25.2.2000

and in revised form on 27.12.2000 